\title{
A Comparative Study between Mixed and Displacement Model and the Effect of the Numerical Integration in the Axisymmetric Shell Finite Element Analysis
}

\author{
H. Djamal, L. Bachir, and Z. Abdallah
}

\begin{abstract}
In this paper the influence of the choice of the model in the formulation of the axisymmetric shell finite element type is presented. A comparison between results obtained by the use of two elements, CAXI_L and CAXI_K is given. The first element is based on standard mixed formulation Hellinger - Reissner and the second one is formulated with displacement model. The variation of the results according to the number of the Gauss integration points is discussed. A Matlab program is elaborated to calculate the displacements for each element.
\end{abstract}

Index Terms-Axisymmetric behavior, formulation model, matlab programming, numerical integration.

\section{INTRODUCTION}

According to shell theory, we can distinguish two types of finite elements:

Finite elements where the transverse shearing is taken into account (Rissner-Mindlin theory).

Finite elements where the effect of the transverse shearing is not taken into account (Love-Kirchoff theory).

Several elements were developed since 1960, the first one formulated (1963) in the field of this type of shell structures is a truncated element which is suitable for revolution shells and is based on Love-Kirchoff theory ([1]-[4]). Currently, the most used element for the analysis of this type of structures is CAXI_K element [5], for this type, the field of displacement $\mathrm{U}$ is linear and $\mathrm{W}$ is cubic. With regard to the elements based on the Rissner-Mindlin, CAXI_L element [5] was proposed and tested. A simple and powerful element based on the displacement model was formulated in [6], the components of $U$ and $\beta$ are linear and $W$ quadratic, and the integration is done with three Gauss points for the membrane, two for the bending and one for the transverse shearing.

The objective of this work is to highlight the influence of the choice of model in the formulation of the finite elements of axisymmetric shell type, thus the influence of the variation of the number of the Gauss points for the numerical integration type; for this, we carried out the development of two programs called Axisym CAXI_L and CAXI_K with Matlab. The first program treats the CAXI_L element, which

Manuscript received May 10, 2013; revised June 28, 2013. A Comparative study between mixed and displacement model and the effect of the numerical integration in the axisymmetric shell finite elements analysis.

The authors are with Civil Engineering and Hydraulics Department Faculty of Sciences and Technology, Biskra University B.P. $07000-$ Algeria (e-mail: d.hamadi@univ-biskra.dz, dhamadiuk@yahoo.com, baclb2007@yahoo.fr, Abdullah@yahoo.fr). is based on Rissner-Mindlin theory, and second is related to the CAXI_K element, which is based on Love-Kirchoff theory.

\section{AXI-SYMETRIC SHELLS THOERY}

\section{A. Love-Kirchoff Theory}

The following assumptions [7] have to be considered: Geometrical assumption of linearization: Displacements and deformations remain small.

Assumption of material linearization: The material obeys the Hook's law

The transverse normal stress is neglected $\sigma_{z}=0$.

The cross-sections, normal in the medium plan not deformed, remain plane and perpendicular to the medium plan deformed $\gamma_{\alpha z}=0, \gamma_{\beta z}=0$ and $\varepsilon_{z}=0$

Displacement model:

The relations efforts resulting-strains are given by:

$$
\begin{aligned}
& {[N]=\left\lfloor H_{m}\right\rfloor\{e\}+\left\lfloor H_{m f}\right\rfloor \chi \chi} \\
& {[M]=\left\lfloor H_{m f}\left\lfloor\{e\}+\left\lfloor H_{f}\right\rfloor \chi \chi\right\}\right.}
\end{aligned}
$$

$N$ : efforts resulting from membrane

$M$ : efforts resulting from bending (moments)

With $\langle e\rangle=\left\langle\begin{array}{ll}e_{s} & e_{\theta}\end{array} \quad\langle\chi\rangle=\left\langle\begin{array}{ll}\chi_{s} & \chi_{\theta}\end{array}\right\rangle\right.$

$e_{s}, e_{\theta}:$ Membrane strains according to $\mathrm{S}$ (meridian) and $\theta$ (circumferential)

$\chi_{s}, \chi_{\theta}$ : Curvatures according to $\mathrm{s}$ and $\theta$.

The displacement model corresponds to (principle of virtual work):

$$
\begin{gathered}
W=W_{\text {int }}-W_{e x t}=0 \\
\left.W_{\text {int }}=2 \pi \int\left(\left\langle e^{*}\right\rangle\left(\left[H_{m}\right]\{e\}+\left[H_{m f}\right](\chi \chi\}\right)+\left\langle\chi^{*}\right\rangle\left(\left[H_{m f}\right] e\right]+\left[H_{f}\right]\{\chi\}\right)\right) r d s
\end{gathered}
$$

$e^{*}, \chi^{*}:$ Membrane strains and virtual curvatures respectively.

\section{B. Rissner Mindlin Theory}

Geometrical assumption of linearization: Displacements is strains remain small.

Assumption of material linearization: The material obeys the law of Hooke

The transverse normal constraint is negligible: $\sigma_{z}=0$ 
Mixed models in transverse shearing

$$
\begin{aligned}
& W=W_{\text {int }}-W_{\text {ext }}=0 \\
& W_{\text {int }}=2 \pi \int_{s}^{\left\langle e^{*}\right\rangle\left(\left[H_{m}\right]\{e\}+\left[H_{m f}\right]\{(\chi\})+\right.}\left\langle\left\langle\chi^{*}\right\rangle\left[H_{m f}\right] e\right]+\left[H_{f}\right]\{(\chi\})+\gamma^{*} T_{S}+T_{s}^{*}\left(\gamma-H_{c}^{-1} T_{S}\right)^{r d s}
\end{aligned}
$$

$T s, T^{*} s$ : real and virtual shearing action followings. Hc : Shearing stiffness.

\section{ELEMENTS FORMULATION}

\section{A. CAXI_LElement}

The finite element CAXI_L [5] is a truncated element with two nodes as presented in Fig.1, its formulation is based on the theory of Reissner-Mindlin. The model used for this element is the mixed model in transverse shearing. We suppose that the shell is discretized by a succession of truncated cones defined by the end nodes on the meridian curve.

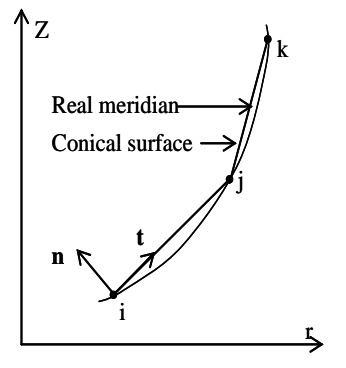

a) discretization

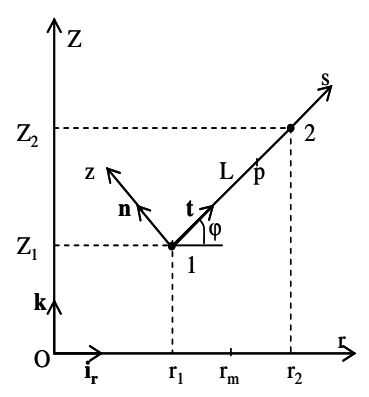

b) Element geometry
Fig. 1. Truncated element CAXI_L (geometry)

The approximations of the displacement field of $\mathrm{U}, \mathrm{W}$ and of $\beta$ are linear in $s$ and $L$ and the shearing action Ts is constant

$$
\begin{gathered}
U=N_{1} U_{1}+N_{2} U_{2} \quad W=N_{1} W_{1}+N_{2} W_{2} \\
\beta=N_{1} \beta_{1}+N_{2} \beta_{2}
\end{gathered}
$$$$
\text { with: } N_{1}=1-s / L \quad ; \quad N_{2}=s / L
$$

The Strains are:

Deformations of membrane $e_{s}, e_{\theta}$

The curvatures are $\chi s, \chi \theta$.

The transverse shearing is $\gamma$

The element stiffness matrix can be evaluated numerically with the reduced integration method of internal work $W^{e}{ }_{i n t}$

$$
\begin{aligned}
& W_{\text {int }}^{e}=\left\langle u_{n}^{*}\right\rangle[K]\left[u_{n}\right\} \\
& \text { With: }[K]=\left\lfloor K_{m f}\right\rfloor+\left\lfloor K_{c}\right\rfloor
\end{aligned}
$$

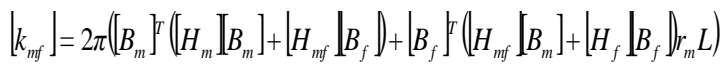

$$
\begin{aligned}
& {\left[k_{c}\right]=\left\{k_{m / T}\right\} \frac{1}{K_{T}}\left\langle k_{m / T}\right\rangle=2 \pi\left\{B_{c}\right\} H_{c} r_{m} L\left\langle B_{c}\right\rangle}
\end{aligned}
$$

with: $\left[k_{c}\right]$ : transverse shearing stiffness matrix.

$\left[k_{m f}\right]$ : membrane bending stiffness matrix for an isotropic material

$$
H_{c}=k . G . h \quad G=E / 2(1+v)
$$

$K=\frac{5}{6}$ (Transverse shearing correction factor)

$$
\begin{gathered}
{\left[H_{m}\right]=\frac{E h}{\left(1-v^{2}\right)}\left|\begin{array}{ll}
1 & v \\
v & 1
\end{array}\right|\left[H_{f}\right]=\frac{E^{3}}{12\left(1-v^{2}\right)}\left|\begin{array}{ll}
1 & v \\
v & 1
\end{array}\right|} \\
{\left[H_{m f}\right]=\frac{E^{3} S}{12\left(1-v^{2}\right) \mathrm{r}_{\mathrm{m}}}\left|\begin{array}{cc}
1 & 0 \\
0 & -1
\end{array}\right|}
\end{gathered}
$$

Resulting efforts (normal effort and bending moment) can be evaluated with (1) and (2)

\section{B. CAXI_K Element}

This finite element is a truncated in shape as shown in Fig. 2. Its formulation based on the Kirchhoff theory [5] and the displacement model. The curvilinear components $U$ (s) and $\mathrm{W}$ (s) are defined by linear approximations and cubic of hermitian type respectively. The numerical integration used is of Gauss type with two points for the evaluation of the stiffness matrix $\left[k^{e}\right]$.
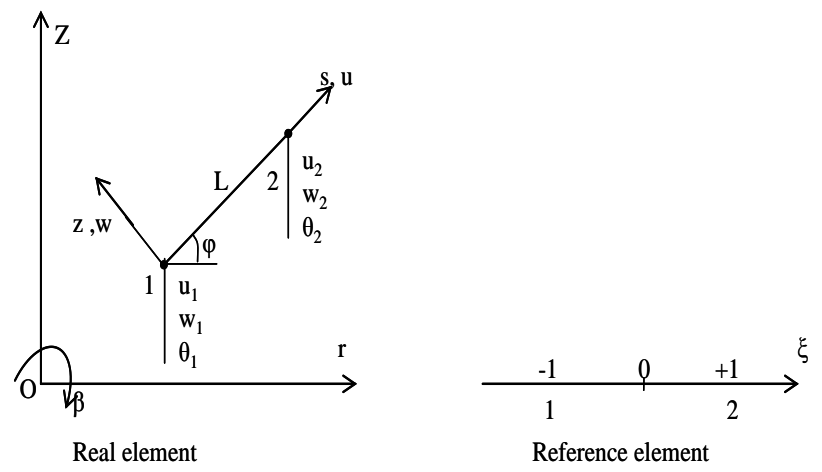

Fig. 2. Truncated element CAXI_K

$$
[k]_{l o c}=2 \pi \int_{s}^{L}\left[k_{\xi}\right] d s=2 \pi \int_{-1}^{1}\left[\mathrm{k}_{\xi}\right] \frac{L}{2} d \xi
$$

with:

$$
\left.k_{\xi}\right\rfloor=\left(\left[B_{m}\right] T\left(\left[\left.H_{m}\right|_{B_{m}}\right]+\left|H_{m f}\right| B_{f} \|+\mid B_{f}\right]^{T}\left(\left.\right|_{H_{m f}}\left[B_{m}\right]+\left|H_{f}\right| B_{f} \mid\right)\right)
$$

The numerical integration according to the Gauss method is:

$$
[k]_{l o c}=2 \pi \sum_{i=1}^{2}\left[k_{\xi}\left(\xi=\xi_{i}\right)\right] \omega_{i} \frac{L}{2}
$$

with

$$
\xi_{i}= \pm 1 / \sqrt{3} \text { and } \omega_{i}=1
$$

After the evaluation of $[k]_{\text {loc, }}$ and before the assembling of the matrices; it is necessary to transform the variables $\left\{u_{n}\right\}_{\text {loc }}$ defined in the local coordinate of the element according to the nodal variables of the cylindrical reference. The transformation matrix $[T]$ is given by: 


$$
\begin{gathered}
{[T]=\left|\begin{array}{cc}
{[t]} & {[0]} \\
0 & 1
\end{array}\right| \quad[t]=\left|\begin{array}{cc}
{[t]^{T}} & 0 \\
{[0]} & {[\mathrm{t}]}
\end{array}\right|} \\
{[Q]=\left[\begin{array}{ll}
t & n
\end{array}\right]=\left|\begin{array}{cc}
C & S \\
S & C
\end{array}\right|}
\end{gathered}
$$

Thus we can write: $\left[k^{e}\right]=[T]^{T}[k]_{l o c}[T]$.

\section{NUMERICAL APPLICATION}

In this section, the presented elements are applied to the analysis of a cylindrical shell as indicate in Fig. 3 fixed at one end is free from the other end. Uniformly distributed load is applied at the free end. The results obtained by the Axisym program for both elements (CAXI_K and CAXI_L), and those of software ANSYS as presented in Fig. 5 are compared with the analytical solution given in [8].

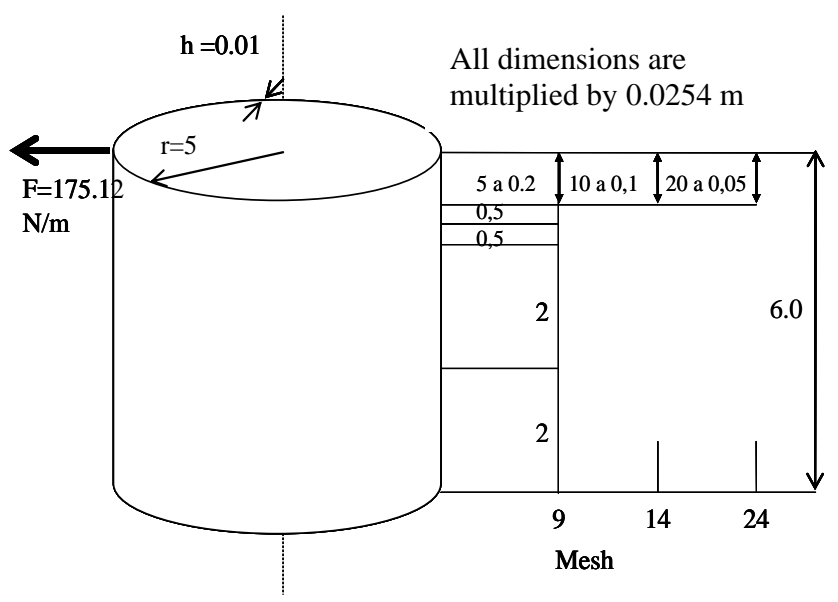

Fig. 3. Cylinder loaded at the free end.

TABLE I: RADIAL DISPLACEMENT (M) AT FREE LOADED END (X0.0254/1000)

\begin{tabular}{|c|c|c|c|c|c|c|}
\hline Mesh & ANSYS & $\begin{array}{c}\text { SOL Ref } \\
{[8]}\end{array}$ & $\begin{array}{c}\text { Prog: } \\
\text { CAXI_K }\end{array}$ & Error & $\begin{array}{c}\text { Prog: } \\
\text { CAXI_L }\end{array}$ & Error \\
\hline 9 & 2,876 & & 2,815 & $2,05 \%$ & 2,875 & $0,03 \%$ \\
\cline { 1 - 4 } 14 & 2,876 & \multirow{2}{*}{2,874} & 2,861 & $0,45 \%$ & 2,875 & $0,03 \%$ \\
\cline { 1 - 1 } 24 & 2,876 & & 2,871 & $0,10 \%$ & 2,875 & $0,03 \%$ \\
\cline { 5 - 6 } & & & & &
\end{tabular}

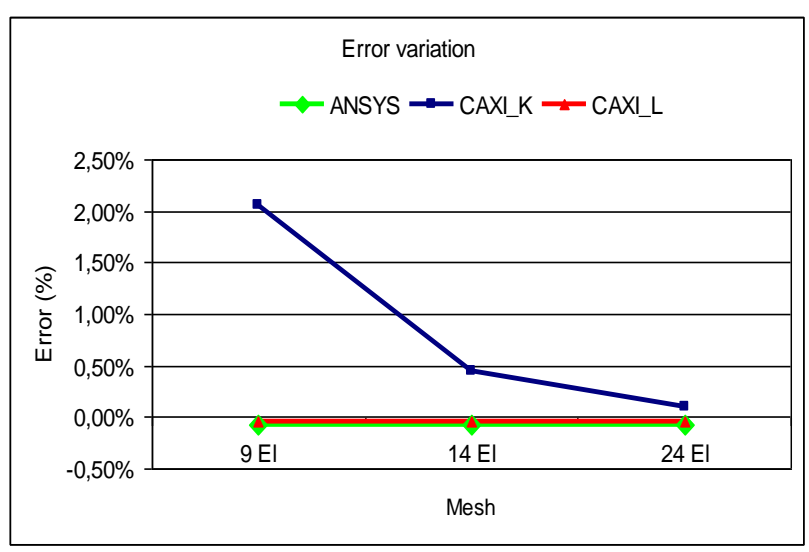

Fig. 4. Convergence of the displacement at the free end
TABLE II: DISPLACEMENT RESULTS ACCORDING TO NUMBER ON INTEGRATION POINTS ( X 0.0254/1000)

\begin{tabular}{|c|c|c|c|c|c|}
\hline \multirow{2}{*}{ Mesh } & $\begin{array}{c}\text { SOL } \\
{[8]}\end{array}$ & $\begin{array}{c}\text { Prog: } \\
\text { CAXI_K } \\
1 \text { pt } \\
\text { integration }\end{array}$ & $\begin{array}{c}\text { Prog: } \\
\text { CAXI_K } \\
3 \text { pts } \\
\text { integration }\end{array}$ & $\begin{array}{c}\text { Prog: } \\
\text { CAXI_K } \\
4 \text { pts } \\
\text { integration }\end{array}$ & $\begin{array}{c}\text { Prog: } \\
\text { CAXI_K } \\
2 \text { pts } \\
\text { integration }\end{array}$ \\
\hline $\begin{array}{c}24 \\
\text { elements }\end{array}$ & 2,874 & 1514 & 2,871 & 2,871 & 0,41 \\
\hline
\end{tabular}

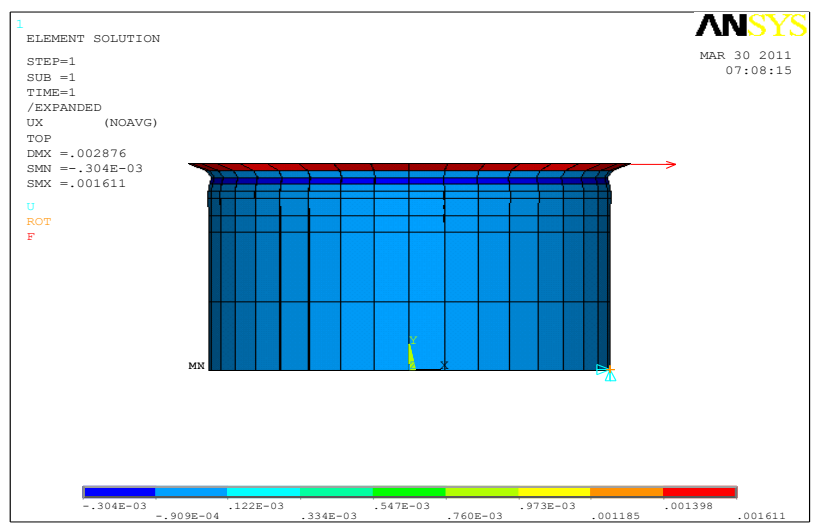

Fig. 5. Deformed structure (ANSYS)

\section{Comments:}

According to the results presented in Table I and Fig.4 for the variation of the error compared to the analytical solution, it can be notice that CAXI_L element gives better results than CAXI_K element.

In Table II we observe that the change of the number of integration points for CAXI_K element does not carry any change on the solution for the displacement even we use a more than 2 points of integration. For the case of one integration point is used, the result will be unacceptable.

For the second element CAXI_L, if we use 2 or more points, the result will be obtained with a great error, therefore only the reduced integration will be applicable.

\section{CONCLUSION}

The choice of the mathematical model leads to very important results in the applications studied; we notice the advantage of the CAXI_L element, which is based on a mixed formulation compared to CAXI_K element based on displacement model formulation, even where for the case where the analytical solution is based on Love-Kirchoff theory, therefore we can say that if the mixed variational formulation is used (standard Hellinger - Reissner), we can develop another mixed model which could give more precision.

For the shell elements, the increases in the numbers of integration points does not carry any improvement in the solution, contrary it gives for some cases bad results, which is noticed in the application for the element CAXI_L. for this case only the integration reduced will be gives good performances. Also if we use a numerical diagram of integration with more than two Gauss points for CAXI_K element, the results will be identical to those given with two points. We should mention that, using only one Gauss point for this type of elements gives unacceptable results. 


\section{REFERENCES}

[1] P. E. Grafton and D. R. Strome, "Analysis of axisymmetric shells by the direct stiffness method," AIAAJ., vol. 1, no. 10, pp. 2342-2347, 1963.

[2] R. R. Meyer and M. B. Harmon, "Conical segment method for analyzing open crown shells of revolution for edge loadings," AIAAJ., vol. 1 , no. 4, pp. 886-891, 1963.

[3] J. H. Percy, T. N. H. Pian, S. Klein, and D. R. Navaratna, "Application of matrix displacement method for linear elastic analysis of shell of revolution," AIAA J., vol. 3, no. 11, pp. 2138-2145, Nov. 1965.

[4] E. P. Popov, J. Penzien, and Z. A. Lu, "Finite element solution for axisymmetric shells," J. Engng. Mech, Div., ASCE, vol. 90, pp. $119-145,1964$

[5] J. L. Batoz and G. Dhatt, "Modélisation des structures par éléments finis, "HERMES, vol. 3, 1992.

[6] A. Tessler, "An efficient conforming axisymmetric shell element including transverse shear and rotary inertia," Computers \& Structures vol. 15, no. 5, pp. 567-574, 1982.

[7] F. Frey and M. A. Sruder, "Analyse des structures en milieu continue," Presses polytechniques et universitaires romandes, vol. 5 , 2003.

[8] C. Rockey, H. R. Evans, D. W. Griffiths, and D. A. Nethercot, "Introduction à la méthode des éléments finis," EYROLLES, 1979.

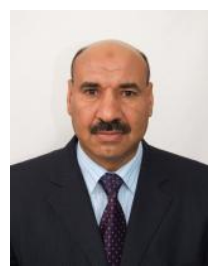

Hamadi Djamal was born in Batna, Algeria, in 1959 He earned M.Sc degree in Civil Eng. Structures, at City University, School of engineering and mathematics, London, United Kingdom, in 1989 and $\mathrm{PhD}$ in Civil Eng. Structures in Biskra in University, Algeria in 2006. He has more than 23 years of teaching experience and currently works as a senior lecturer at the civil and hydraulics department Faculty of Sciences and Technology, and a researcher in the Laboratory of Civil Engineering,
Hydraulics, Development and Durability, Biskra University, Algeria.

Dr. Djamal has published more than 20 research papers in International association of Engineering conference proceedings and international journals. His research interests include numerical analysis of structures, finite element method, strain based approach, structures modeling and FORTRAN programming finite elements.

Dr. Hamadi became a Member of IAENG since 2008.

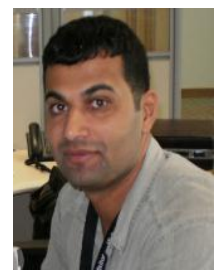

Labiodh Bachir was born in October 1975 in Algeria. and getting her first degree, civil engineer in USTHB Algiers in October 1999. Then he continued deeply in Master of modeling materials and Structures at Biskra University and currently his pursuing in $\mathrm{PhD}$ level at Biskra University in study of shell structures.

Mr. Bachir has published 2 research papers in International Engineering conference. His research interests include structures modeling, numerical analysis of structures, finite element method, programming (finite element)

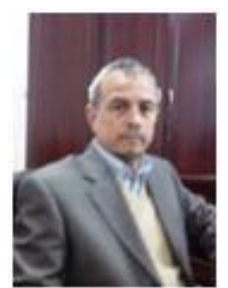

Zatar Abdallah has more than 27 years of teaching experience and currently works as Vice Director of Civil and Hydraulics Department and a senior lecturer at Faculty of Sciences and Technology, and a researcher in the Laboratory of Civil Engineering, Biskra University, Algeria.

Dr. Abdallah has published more than 15 research papers in International Engineering conference proceedings and international journals. His research interests include computer aid design, finite element method and local materials. 